Det er mi nat $i$ on of the hydr ogen and hel $i$ um i on densities in the initial and final stages of a pl asma i n the Large Hel i cal Devi ce by opt i cal spect r oscopy

\begin{tabular}{|l|l|}
\hline $\begin{array}{l}\text { jour nal or } \\
\text { publ } \mathrm{i} \text { cat } \mathrm{i} \text { on } \mathrm{ti} \mathrm{tl} \text { e }\end{array}$ & Physi cs of Pl asmas \\
\hline vol une & Vol . 10 \\
\hline number & I ssue 5 \\
\hline page $\mathrm{r}$ ange & pp. 1402-1410 \\
\hline year & 2003- 05- 01 \\
\hline URL & ht t p: //hdl . handl e. net /10655/2218 \\
\hline
\end{tabular}




\title{
Determination of the hydrogen and helium ion densities in the initial and final stages of a plasma in the Large Helical Device by optical spectroscopy
}

\author{
M. Goto and S. Morita \\ National Institute for Fusion Science, Toki 509-5292, Japan \\ K. Sawada \\ Department of Applied Physics, Faculty of Engineering, Shinshu University, Nagano 380-8553, Japan \\ T. Fujimoto \\ Department of Engineering Physics and Mechanics, Graduate School of Engineering, Kyoto University, \\ Kyoto 606-8501, Japan \\ S. Yamamoto \\ Department of Energy Engineering and Science, Nagoya University, Nagoya 464-8601, Japan \\ J. Miyazawa, H. Yamada, and K. Toi \\ National Institute for Fusion Science, Toki 509-5292, Japan
}

(Received 10 October 2002; accepted 11 February 2003)

\begin{abstract}
Emission line intensities in the UV and visible wavelength range have been measured in the initial and final stages of a discharge in the Large Helical Device (LHD) [O. Motojima et al., Phys. Plasmas 8, 1843 (1999)]. From these results combined with $T_{e}$ and $n_{e}$, which are determined spectroscopically, the ionization and recombination fluxes of hydrogen and helium are estimated in the region of high line emissivities. The temporal behavior of line-averaged $n_{e}$ which is evaluated from these fluxes is found to be consistent with the results of the interferometer measurement. This implies that in such transient stages of the plasma the temporal variation of $n_{e}$ is dominated by the atomic ionization-recombination processes rather than the particle transport or diffusion. On the basis of that characteristic in LHD, the line-averaged ion densities for hydrogen and helium just before the termination of the plasma heating are determined. Thus, a method of the density determination of the fueling gas and of the ash is suggested for future fusion devices. (c) 2003
\end{abstract} American Institute of Physics. [DOI: 10.1063/1.1566024]

\section{INTRODUCTION}

With the help of collisional-radiative (CR) model calculations, we have shown in a previous article that an emission line intensity serves as a measure of the ionization flux or the recombination flux of atoms or ions so that the magnitude of these fluxes can be estimated spectroscopically. ${ }^{1}$ In the same article, we have presented the proportionality factors between some representative emission lines and the ionization and the recombination fluxes for neutral hydrogen and neutral and ionized helium, which are the important constituents of fusion plasmas. In the present study we extend our work to establish a method of determining the hydrogen and helium ion densities from the ionization and recombination fluxes for these atoms and ions.

As the target of this attempt we took a plasma in the Large Helical Device (LHD), which is a heliotron type device for fusion study. ${ }^{2}$ In LHD, the magnetic field for plasma confinement is externally given and essentially steady even in the transient state such as the initial and final stages of discharges, so that the temporal variation of the electron density or the ion density is considered to be dominated by the atomic ionization and recombination processes, at least in a short time scale. Thus, the LHD plasma is an ideal light source to realize our objective.
In LHD it has been known that a significant amount of helium is mixed into the plasma, since glow discharge cleaning (GDC) with helium gas is performed before the main discharges; this is necessary because of the relatively low baking temperature $\left(90^{\circ} \mathrm{C}\right)$ of the vacuum vessel wall. However, a quantitative measurement of the amount of helium ions has not been done. Meanwhile, in the experiment of the ion cyclotron heating (ICH), the minority proton heating regime is achieved using helium ions instead of deuterons. ${ }^{3}$ Since the heating efficiency is known to depend on the ion density ratio of hydrogen to helium, an accurate ion density ratio is needed. Our method is found to be useful for these purposes. In the final section, we present the result of our attempt to estimate the individual ion densities for hydrogen and helium.

\section{EXPERIMENTAL SETUP}

For the present experiment, the nominal magnetic field strength, $B_{\mathrm{ax}}$, and the radius of the magnetic axis, $R_{\mathrm{ax}}$, are $2.64 \mathrm{~T}$ and $3.75 \mathrm{~m}$, respectively. Helium gas is introduced into the vacuum vessel to the background pressure of the order of $10^{-3} \mathrm{~Pa}$. A discharge is started with the electron cyclotron heating $(\mathrm{ECH})$ and the plasma is sustained with the neutral beam injection (NBI). After the ignition of the 


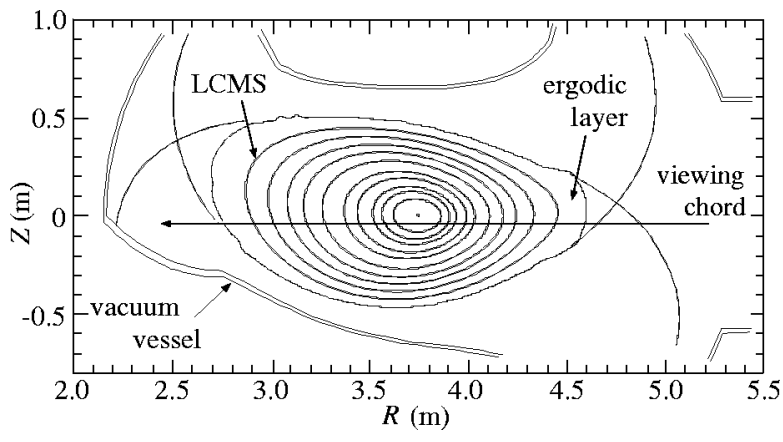

FIG. 1. Magnetic surfaces for the configuration of $R_{\mathrm{ax}}=3.75 \mathrm{~m}$ at the cross section for the present observation. The $R$ and $Z$ axes indicate the major radius direction and the direction perpendicular to the equatorial plane, respectively. The viewing chord is indicated with an arrow.

plasma, additional helium gas is supplied by gas-puffing and the plasma density is increased. Though only helium gas is introduced into the vacuum vessel, a significant amount of hydrogen is found to be mixed into the plasma; this is due to the NBI and also to recycling from the wall.

In the initial and final stages of the discharge, spectra in the UV and visible wavelength range are observed. The viewing chord almost coincides with the major axis of the elliptical poloidal cross section of the plasma elongated in the major radius direction, as shown in Fig. 1. The field of view is collimated with a lens so that the spatial resolution is about $30 \mathrm{~mm}$. The collected light is guided with an optical fiber having $100 \mu \mathrm{m}$ diameter to a $50 \mathrm{~cm}$ Czerny-Turnertype spectrometer (Chromex 500is) equipped with a 150 grooves/mm grating. A CCD (charge coupled device: Andor DU-420UV) is used as the detector and the wavelength range of $200 \mathrm{~nm}-550 \mathrm{~nm}$ is measured simultaneously.

For the measurement the so-called "fast kinetic mode" of the detector is utilized, in which only a narrow layer on the CCD detector surface is exposed and the accumulated electric charges are sequentially shifted with a designated shifting rate. The shifted charges are stored in the remaining area of the CCD and read out as the storage area becomes full. In the following measurement the time duration of about $1.2 \mathrm{~s}$ for each of the initial stage and the final stage of the discharge is measured with a sampling rate of 200 spectra per second.

The absolute sensitivity of the observation system has been calibrated with a standard tungsten lamp (Eppley Laboratory) and a standard deuterium lamp (Optronic Laboratories) in combination with a diffuser plate made of the "white reflectance cating" (KODAK 6080) which has a reflection efficiency of higher than $93 \%$ in the wavelength range of longer than $250 \mathrm{~nm}$. We observed the reflected light from the plate irradiated by each of the standard lamps with the same observation system as used in the actual measurement. Thus, the sensitivity of the system was determined.

\section{COLLISIONAL-RADIATIVE MODEL}

According to the idea of the CR model, the excited level population, $n(p)$, of level $p$ is expressed as a summation of two terms as

$$
n(p)=R_{0}(p) n_{e} n_{i}+R_{1}(p) n_{e} n_{0},
$$

where $R_{0}(p)$ and $R_{1}(p)$ are functions of $n_{e}$ and $T_{e}$, and called the population coefficients. $n_{0}$ is the ground state density of the atom or the ion under consideration, and $n_{i}$ is the density of the next ionized stage ion. The first and second terms are called the recombining plasma component and the ionizing plasma component, respectively. The photon emission rate of the $p \rightarrow q$ transition, $A(p, q) n(p)$, where $A(p, q)$ is the spontaneous transition probability, is written as

$$
\begin{aligned}
A(p, q) n(p) & =A(p, q)\left[R_{0}(p) n_{e} n_{i}+R_{1}(p) n_{e} n_{0}\right] \\
& =\varepsilon_{0} n_{e} n_{i}+\varepsilon_{1} n_{e} n_{0} .
\end{aligned}
$$

Here, $\varepsilon_{0}$ and $\varepsilon_{1}$ are called the photon emission rate coefficients. The temporal developments of $n_{0}$ and $n_{i}$ are expressed in the CR model as

$$
\frac{\mathrm{d} n_{0}}{\mathrm{~d} t}=-\frac{\mathrm{d} n_{i}}{\mathrm{~d} t}=-S_{\mathrm{CR}} n_{e} n_{0}+\alpha_{\mathrm{CR}} n_{e} n_{i},
$$

where $S_{\mathrm{CR}}$ and $\alpha_{\mathrm{CR}}$ are called the CR ionization and recombination rate coefficients, respectively.

From here, we take hydrogen as an example and consider the ionizing plasma and the recombing plasma, separately. In the ionizing plasma, the time derivative of the proton density, $\mathrm{d} n_{\mathrm{H}^{+}} / \mathrm{d} t$, is expressed as

$$
\frac{\mathrm{d} n_{\mathrm{H}^{+}}}{\mathrm{d} t}=S_{\mathrm{CR}} n_{e} n_{\mathrm{H}^{0}},
$$

where $n_{\mathrm{H}^{0}}$ is the ground state density of neutral hydrogen. On the other hand, the line intensity, $I_{\mathrm{H}}$, is defined as the photon emission rate of the ionizing plasma component, and is written as

$$
I_{\mathrm{H}}=\varepsilon_{1} n_{e} n_{\mathrm{H}^{0}} .
$$

As a result, Eq. (5) is rewritten as

$$
\frac{\mathrm{d} n_{\mathrm{H}^{+}}}{\mathrm{d} t}=\kappa_{1} I_{\mathrm{H}},
$$

where $\kappa_{1}$ stands for $S_{\mathrm{CR}} / \varepsilon_{1}$.

In the recombining plasma, $\mathrm{d} n_{\mathrm{H}^{+}} / \mathrm{d} t$ is expressed as

$$
\frac{\mathrm{d} n_{\mathrm{H}^{+}}}{\mathrm{d} t}=-\alpha_{\mathrm{CR}} n_{e} n_{\mathrm{H}^{+}} .
$$

The line intensity of neutral hydrogen in a recombining plasma is expressed as

$$
I_{\mathrm{H}}=\varepsilon_{0} n_{e} n_{\mathrm{H}^{+}},
$$

and Eq. (8) is rewritten as

$$
\frac{\mathrm{d} n_{\mathrm{H}^{+}}}{\mathrm{d} t}=-\kappa_{0} I_{\mathrm{H}},
$$

where $\kappa_{0}$ stands for $\alpha_{\mathrm{CR}} / \varepsilon_{0}$.

The reliability of the CR model code naturally depends on the accuracy of the atomic data in it. The electron impact excitation cross sections are most critical and we took special care and adopted the best available data at present. For neutral hydrogen the code has been developed by Sawada. ${ }^{4}$ The excitation cross section data in it are based on Johnson's 


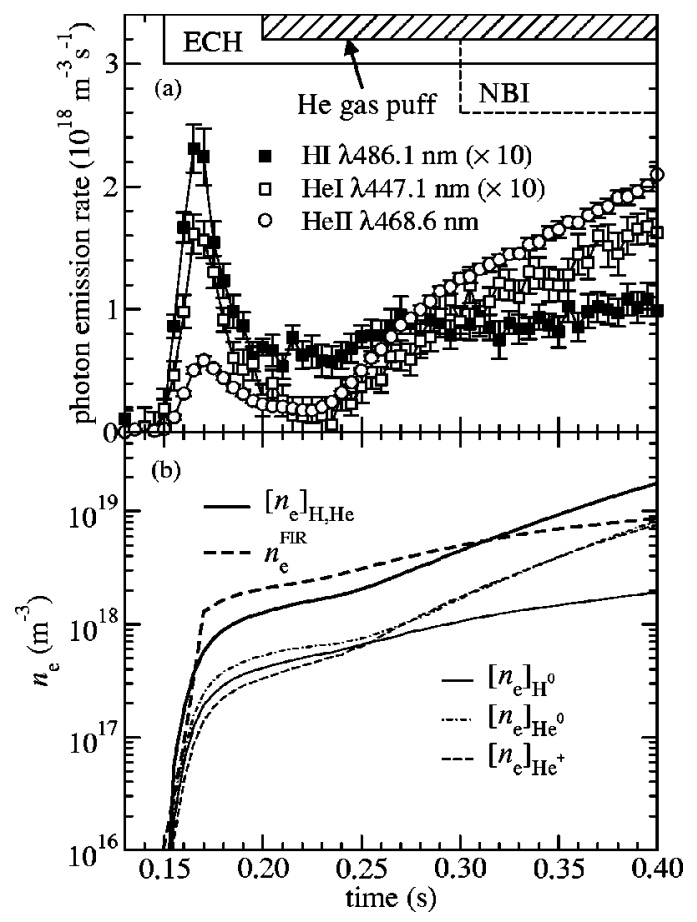

FIG. 2. Temporal variations of (a) the photon emission rates of the HI $\lambda$ $486.1 \mathrm{~nm}$, HeI $\lambda 447.1 \mathrm{~nm}$, and HeII $\lambda 468.6 \mathrm{~nm}$ lines and (b) contributions to the electron density from neutral hydrogen, $\left[n_{e}\right]_{\mathrm{H}^{0}}$, neutral helium, $\left[n_{e}\right]_{\mathrm{He}^{0}}$, and ionized helium, $\left[n_{e}\right]_{\mathrm{He}^{+}}$in the initial stage of the discharge. For the latter, summation of all the contributions, $\left[n_{e}\right]_{\mathrm{H}, \mathrm{He}}$, and the lineaveraged electron density measured with the interferometer, $n_{e}^{F I R}$, are also shown.

semiempirical formula ${ }^{5}$ and the parameters in the formula are adjusted to give the best fit with the other available theoretical and experimental data. For neutral helium, the code which was originally constructed by Fujimoto ${ }^{6}$ and recently revised by Goto $^{7}$ is used. In this code the excitation cross section data that are calculated with the convergent close coupling (CCC) method ${ }^{8-10}$ and are parametrized for practical use ${ }^{11}$ are adopted. For ionized helium, the COLRAD $\operatorname{code}^{12,13}$ designed for general hydrogenlike ions is employed, but we have improved the excitation cross section data in it ${ }^{1}$ by replacing them with the Fisher's semi-empirical data. ${ }^{14}$ The COLRAD code has an option with which the fine structure levels up to $n=4$, where $n$ is the principal quantum number, are treated separately, but here we do not use this option and consider only the $n$-resolved levels up to $n=50$.

\section{RESULTS}

\section{A. Initial stage-ionizing plasma}

Figure 2(a) shows the temporal variations of the emission intensities of the HI $\lambda 486.1 \mathrm{~nm}(n=2-4)$, HeI $\lambda 447.1$ $\mathrm{nm}\left(2{ }^{3} P-4{ }^{3} D\right)$, and HeII $\lambda 468.6 \mathrm{~nm}(n=3-4)$ lines. The measured line intensities, which are line-integrated along the viewing chord, are divided by the chord length $(\sim 1.8 \mathrm{~m})$ to give line-averaged values. The $\mathrm{ECH}$ injection with resonance near the magnetic axis starts at $t=0.15 \mathrm{~s}$ and the NBI is superposed from $t=0.3 \mathrm{~s}$. The gas-puffing of helium starts at $t=0.2 \mathrm{~s}$. The line emissions appear instantaneously with the ECH injection and almost quench in a moment. Though no hydrogen gas is supplied into the plasma at this moment, a rather strong line emission of neutral hydrogen is observed. This hydrogen emission is assumed to come from the residual hydrogen gas in the vacuum vessel. As the main gaspuffing of helium is started, the intensities of helium lines increase again.

Since the measured signal is line integrated, there remains an ambiguity about the position of the emission region. In fact, we have 40 parallel viewing chords including the one used in the present measurement ${ }^{15}$ and they cover the entire plasma cross section in Fig. 1. The spectra from all the chords can be simultaneously measured with a lower sampling rate, e.g., $5 \mathrm{~Hz}$. In such measurements, we have confirmed that the emission region starts from the central region and expands in the initial stage, and in the final stage it shrinks to the central region. In addition to that, we have measured the Zeeman splittings of emission lines as mentioned later, and they indicate the emission region resides near the magnetic axis in these stages of a discharge.

In the initial stage of the present discharge, the emission line intensity reflects the ionization flux, ${ }^{1}$ or in other words, the electron production rate of the respective atoms or ions. Owing to the externally produced steady-state magnetic field in LHD, electrons produced in the confinement region are expected to be accumulated in the plasma. If this is the case, the line intensity indicates the rate of increase in the electron density. Indeed, the 11-chord far-infrared (FIR) interferometer system ${ }^{16}$ indicates the electron density grows up from the central region.

In order to simplify the consideration, we assume here a homogeneous plasma. By integrating Eq. (7), we obtain

$$
n_{\mathrm{H}^{+}}(t)=\left[n_{e}\right]_{\mathrm{H}^{0}}(t)=\int_{0}^{t} \kappa_{1}\left(t^{\prime}\right) I_{\mathrm{H}}\left(t^{\prime}\right) \mathrm{d} t^{\prime},
$$

where $\left[n_{e}\right]_{\mathrm{H}^{0}}$ is the contribution from neutral hydrogen to $n_{e}$. Figure 3(a) shows the dependences of $\kappa_{1}$ on $T_{e}$ and $n_{e}$. The $T_{e}$ and $n_{e}$ values necessary to determine $\kappa_{1}$ are evaluated from the line intensity ratios of neutral helium ${ }^{7,17,18}$ for a different but similar discharge. The results are shown in Fig. 4. For the density, the line-averaged result, which is measured on the center chord of the interferometer system, $n_{e}^{F I R}$, is also shown and it is found that in the time region $t<0.3 \mathrm{~s}$ both the results agree well and later they deviate from each other. It should be remembered here that the interferometer measurement gives the line-averaged values along the viewing chord, while our results give the parameters at the location of the dominant atom emissions. The discrepancy can be understood from this. Their good agreement for $t<0.3 \mathrm{~s}$ indicates that the atom emissions and ionization processes take place in the same central plasma region. The subsequent discrepancy between these data suggests that those atomic processes take place in the plasma edge region, which is likely outside the last closed magnetic surface (LCMS). ${ }^{15}$ Most of the electrons and ions produced outside the LCMS are thought to be immediately trapped by the magnetic field and led to the divertor plates without contributing to the density increase of the main plasma. ${ }^{19} \mathrm{We}$ must bear this fact in mind. Anyway, since our interest is 


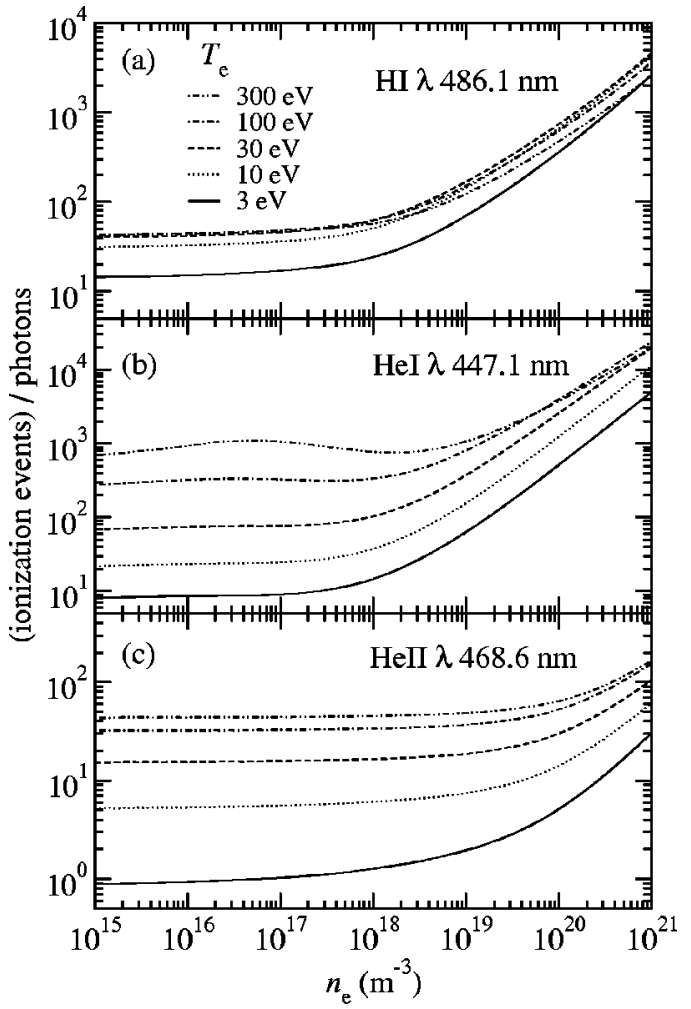

FIG. 3. Dependences of $\kappa_{1}=S_{\mathrm{CR}} / \varepsilon_{1}$ on $T_{e}$ and $n_{e}$ for (a) the HI $\lambda 486.1$ nm, (b) HeI $\lambda 447.1 \mathrm{~nm}$, and (c) HeII $\lambda 468.6 \mathrm{~nm}$ lines.

limited to the estimation of the ionization flux from the emission line intensity, the $T_{e}$ and $n_{e}$ values obtained from the helium lines are more appropriate.

We determine $\kappa_{1}$ from Fig. 3(a) with the obtained $T_{e}$ and $n_{e}$ values, and evaluate the right-hand side of Eq. (11). Similar analyses for neutral and ionized helium are also carried out with the $\kappa_{1}$ values shown in Figs. 3(b) and 3(c). Figure 2(b) shows their contributions to the electron density which are designated as $\left[n_{e}\right]_{\mathrm{H}^{0}},\left[n_{e}\right]_{\mathrm{He}^{0}}$, and $\left[n_{e}\right]_{\mathrm{He}^{+}}$. The summation of all the components, $\left[n_{e}\right]_{\mathrm{H}, \mathrm{He}}$, that corresponds to the contributions from hydrogen and helium to the total electron density is shown with the thick solid line in Fig. 2(b). In the same figure $n_{e}^{F I R}$ is also shown. If the plasma is homogeneous, $\left[n_{e}\right]_{\mathrm{H}, \mathrm{He}}$ is directly comparable with $n_{e}^{F I R}$, but such an assumption is not substantiated for real plasmas even in the time range where the ionization processes take place in the main plasma region. It is, however, worth mentioning that since the viewing chord of the interferometer is geometrically similar to ours, the comparison is still meaningful. In Fig. 2(b) it is readily noticed that the temporal behaviors of both the results are similar in the time region $t<0.25 \mathrm{~s}$. Though the absolute values are different by a factor of about 2 , we judge the agreement is satisfactory considering the facts that the geometries are not identical and the influences of impurity ions are completely ignored in the present analysis. In the later time period the rate of increase in $\left[n_{e}\right]_{\mathrm{H}, \mathrm{He}}$ is larger than $n_{e}^{F I R}$; this is probably because the electrons produced in the dominant emission region of hydrogen and helium no longer contribute to the increase in the electron density of the well-confined region.

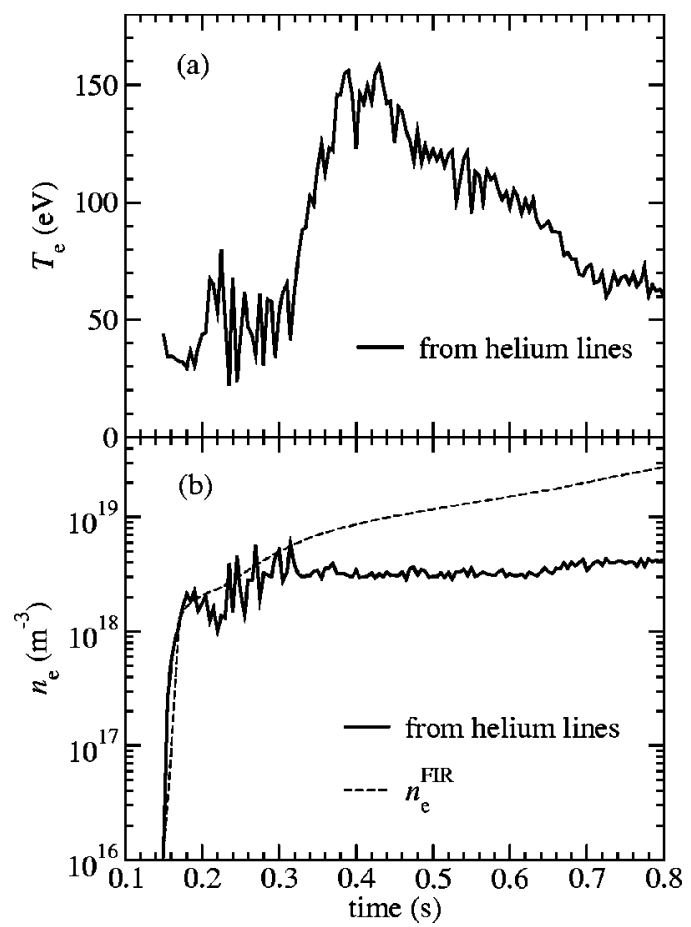

FIG. 4. Temporal variations of (a) $T_{e}$ and (b) $n_{e}$ in the initial stage of the discharge deduced from the line intensity ratios of neutral helium. The interferometer measurement, $n_{e}^{F I R}$, is also shown in (b).

It is worth pointing out that, in the time region of $t$ $>0.3$ s, the rates of increase in $\left[n_{e}\right]_{\mathrm{He}^{0}}$ and $\left[n_{e}\right]_{\mathrm{He}^{+}}$coincide. This indicates the same magnitudes of the ionization flux of neutral helium and that of ionized helium. In the parameter ranges of this time region, the effective ionization time of ionized helium is estimated to be about $10^{-4} \mathrm{~s}$ from calculation with the COLRAD code. According to Ref. 15 most of the neutral helium is ionized outside the LCMS, and its atom temperature is about $300 \mathrm{~K}$ which corresponds to a thermal velocity of $1.4 \times 10^{3} \mathrm{~m} / \mathrm{s}$. These values suggest that $\mathrm{He}^{+}$ions move about $10 \mathrm{~cm}-20 \mathrm{~cm}$ before being ionized once more. On the other hand, when the produced helium ions move along the magnetic field line, the distance to the divertor plates is in the range of $10 \mathrm{~m}-10^{3} \mathrm{~m}$ (Ref. 20). Therefore it is expected that though helium is usually first ionized outside the LCMS, it has enough time to be fully ionized before finally arriving at the divertor plates. The observed identical ionization fluxes of neutral and ionized helium are thus explained.

We have used throughout the plasma parameters measured with the spectroscopic method, and the electron temperature has not been cross-checked with other measurements. Here, we estimate the influence of the error of the electron temperature on the final result. In the measurement of the electron temperature, the strong $T_{e}$ dependence of the line intensity ratio between the $2{ }^{1} P-3{ }^{1} S$ and $2{ }^{3} P-3{ }^{3} S$ lines are exploited. Since the density is relatively low, the populations of the upper levels of these transitions, $3{ }^{1} S$ and $3{ }^{3} S$, are mainly formed by the electron impact excitation from the ground state and the reliability of the result heavily depends on the cross section data used in the CR model code. For these transitions the CCC data incorporated in the 


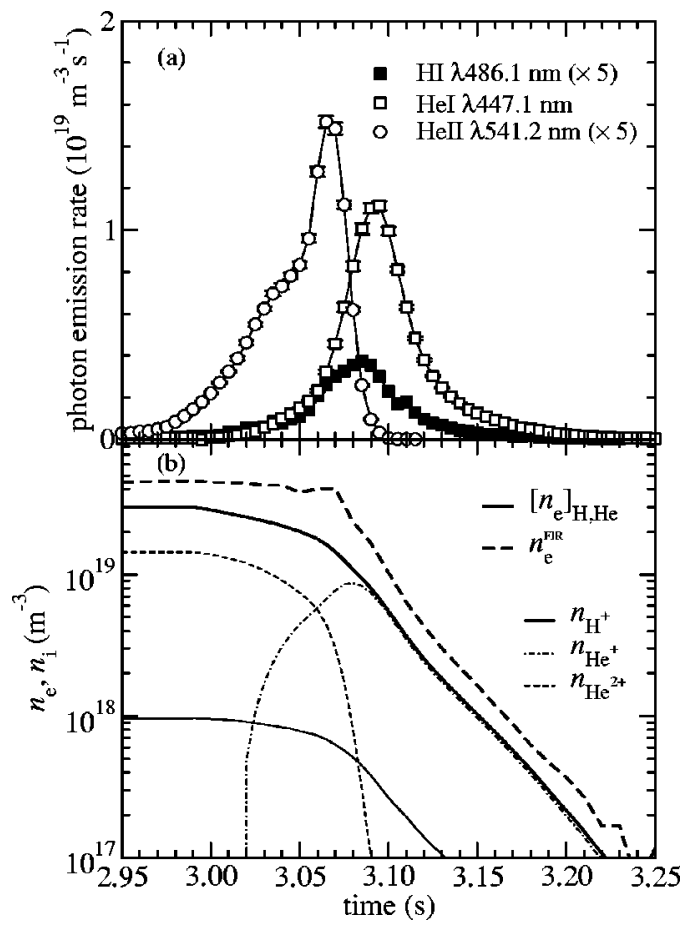

FIG. 5. Temporal variations of (a) the intensity for HI $\lambda 486.1 \mathrm{~nm}, \mathrm{HeI} \lambda$ $447.1 \mathrm{~nm}$, and HeII $\lambda 541.2 \mathrm{~nm}$ lines and (b) the evaluated ion densities, $n_{\mathrm{H}^{+}}, n_{\mathrm{He}^{+}}$, and $n_{\mathrm{He}^{2+}}$ in the final stage of the discharge. For the latter, summation of the contributions to the electron density from hydrogen and helium, $\left[n_{e}\right]_{\mathrm{H}, \mathrm{He}}$, and the interferometer measurement, $n_{e}^{F I R}$, are also shown.

code are known to agree well with other theoretical and experimental data, and are considered to be highly reliable. In the time region of $t<0.3 \mathrm{~s}$, the obtained $T_{e}$ values have a relatively large uncertainties due to weak signals. However, even if $T_{e}$ is changed by $50 \%$, the estimated ionization flux is changed by less than $10 \%$ for neutral hydrogen and less than $50 \%$ for neutral and ionized helium. In this time region the contributions from these three species to $\left[n_{e}\right]_{\mathrm{H}, \mathrm{He}}$ are similar, and hence the uncertainty of $\left[n_{e}\right]_{\mathrm{H}, \mathrm{He}}$ is at largest about $30 \%$. This might be one of the reasons for the discrepancy between $\left[n_{e}\right]_{\mathrm{H}, \mathrm{He}}$ and $n_{e}^{F I R}$ in Fig. 2(b).

\section{B. Final stage-recombining plasma}

In the final stage of a discharge, the emission lines of ionized helium terminating on $n=3$ and $n=4$ levels are found to be rather strong. Since the wavelengths of the Balmer series lines of neutral hydrogen are so close to those of the ionized helium lines for the $n=4-p$ transitions, where $p$ is even, that an independent measurement of the hydrogen Balmer series lines is impractical with the observation system employed here. However, as mentioned later the intensity of the HI $\lambda 486.1 \mathrm{~nm}$ line, for example, can be estimated as the difference between the measured and the theoretically expected intensities of the HeII $(n=4-8)$ line.

Figure 5(a) shows the temporal variations of the emission intensities for the HI $\lambda 486.1 \mathrm{~nm}, \mathrm{HeI} \lambda 447.1 \mathrm{~nm}$, and HeII $\lambda 541.2 \mathrm{~nm}(n=4-7)$ lines in the final stage of the same discharge as the one analyzed in the previous section. The reason why the $\lambda 541.2 \mathrm{~nm}$ line is used for ionized

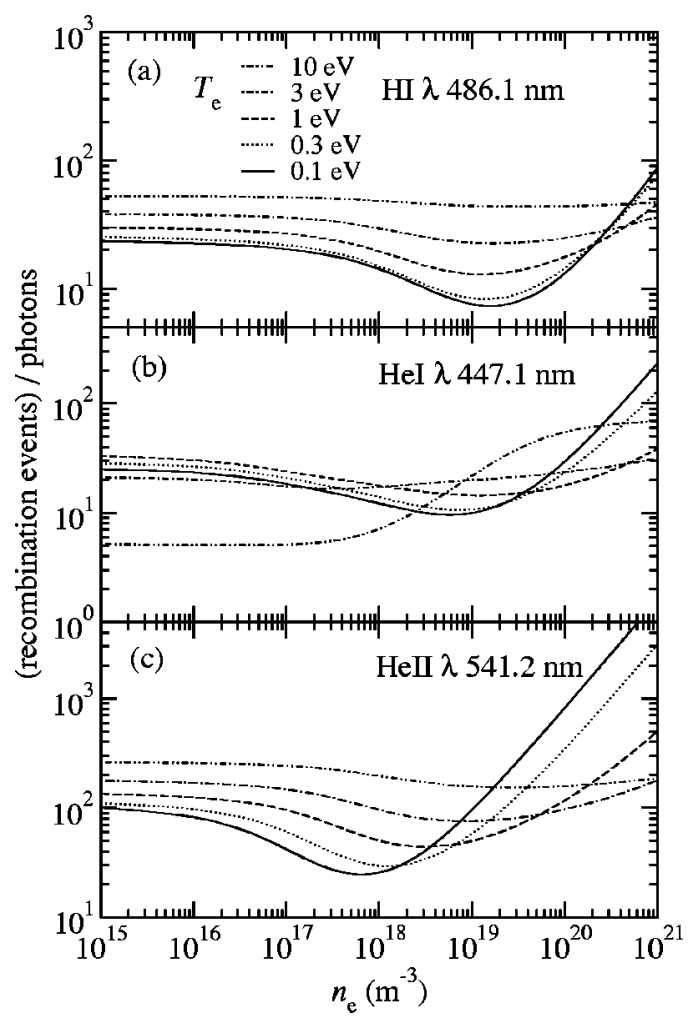

FIG. 6. Dependences of the $\kappa_{0}=\alpha_{\mathrm{CR}} / \varepsilon_{0}$ on $T_{e}$ and $n_{e}$ for (a) the HI $\lambda$ $486.1 \mathrm{~nm}$, (b) HeI $\lambda 447.1 \mathrm{~nm}$, and (c) HeII $\lambda 541.2 \mathrm{~nm}$ lines.

helium instead of the $\lambda 468.6 \mathrm{~nm}$ line, which was used for the ionizing plasma case, is explained later. Though the NBI heating is terminated at $t=2.8 \mathrm{~s}$, the electron density is found to be kept almost unchanged during about $200 \mathrm{~ms}$. The intensity peaks of the neutral lines delayed a little as compared with the ionized helium line and decay rather slowly.

In this case the line intensity reflects the recombination flux of the respective atoms or ions. ${ }^{1}$ Here, we consider the temporal variation of the ion density assuming a homogeneous plasma again. By integrating Eq. (10), we obtain $n_{\mathrm{H}^{+}}$ as a function of time as

$$
n_{\mathrm{H}^{+}}(t)=\int_{t}^{\infty} \kappa_{0}\left(t^{\prime}\right) I_{\mathrm{H}}\left(t^{\prime}\right) \mathrm{d} t^{\prime},
$$

where $n_{\mathrm{H}^{+}}=0$ for $t \rightarrow \infty$ is used. A similar consideration is valid for $\mathrm{He}^{2+}$ and $\mathrm{He}^{+}$, and their densities, $n_{\mathrm{He}^{2+}}$ and $n_{\mathrm{He}^{+}}$, are respectively expressed as

$$
n_{\mathrm{He}^{2+}}(t)=\int_{t}^{\infty} \kappa_{0}\left(t^{\prime}\right) I_{\mathrm{He}^{+}}\left(t^{\prime}\right) \mathrm{d} t^{\prime},
$$

and

$$
n_{\mathrm{He}^{+}}(t)=\int_{t}^{\infty} \kappa_{0}\left(t^{\prime}\right) I_{\mathrm{He}}\left(t^{\prime}\right) \mathrm{d} t^{\prime}-n_{\mathrm{He}^{2+}}(t),
$$

where $I_{\mathrm{He}}$ and $I_{\mathrm{He}^{+}}$are the line intensities of neutral and ionized helium, respectively.

The dependences of $\kappa_{0}$ on $T_{e}$ and $n_{e}$ for the present three emission lines are shown in Fig. 6. The plasma parameters necessary to determine $\kappa_{0}$ 's are estimated from the population distribution over the excited levels of $\mathrm{He}^{+}$and 


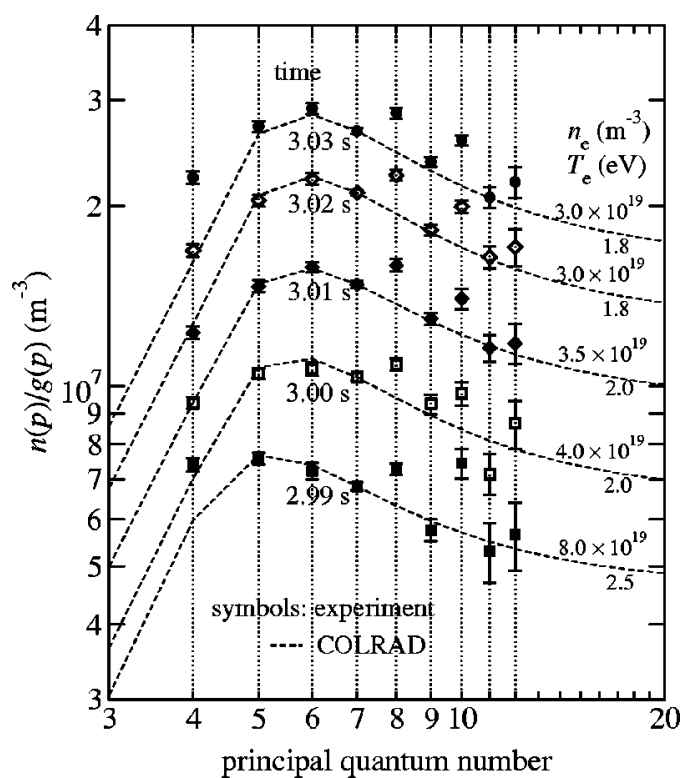

FIG. 7. Temporal variations of the excited level populations divided by the statistical weights for $\mathrm{He}^{+}$obtained in the final stage of the discharge (symbols). Also shown are the $T_{e}$ and $n_{e}$ values estimated with the help of the $\mathrm{CR}$ model code, and the calculation results of the populations with the obtained parameters which are normalized to the experimental data for the $n=7$ level (dashed lines).

$\mathrm{He}^{0}$. Figure 7 shows the temporal variation of the populations over the several excited levels for $\mathrm{He}^{+}$that is divided by the statistical weights. The populations of the levels $n$ $=4$ to $n=6$ are deduced from the line intensities of the transitions terminating on $n=3$ and others are deduced from those terminating on $n=4$. The apparent population densities for the $n=8,10$, and 12 levels are found rather large as compared with those of the other levels. This is interpreted as due to the blended Balmer series lines of neutral hydrogen. With the help of calculations with the COLRAD code, $T_{e}$ and $n_{e}$ values are obtained so as to obtain the best fit of the calculated population distribution to the measured one except the $n=8,10$, and 12 levels.

The $n=4$ level density cannot be fitted with any reasonable parameters. There are several possibilities for this, e.g., an influence of the ionizing plasma component, a contribution from the charge exchange recombination processes with excited hydrogen atoms, and so on, but the reason is still unclear. Roughly speaking, $T_{e}$ is determined from the slope of the populations in the highly excited levels, for which the Saha-Boltzmann distribution ${ }^{21}$ is a good approximation and $n_{e}$ is determined from their distribution profile near the peak level of population. Considering this procedure of the parameter determination, we conclude that even if we disregard the discrepancy in the $n=4$ level population between the experimental and calculation results, the deduced parameters are accurate enough for the present purpose. This fact suggests that the intensity of the HeII $\lambda 468.6 \mathrm{~nm}(n=3-4)$ line is inappropriate to be used in the following analyses and we judge the HeII $\lambda 541.2 \mathrm{~nm}(n=4-7)$ line to be more preferable. The obtained $T_{e}$ and $n_{e}$ and the corresponding population distribution profile normalized to the level $n=7$ are shown in Fig. 7. The difference between the measured and
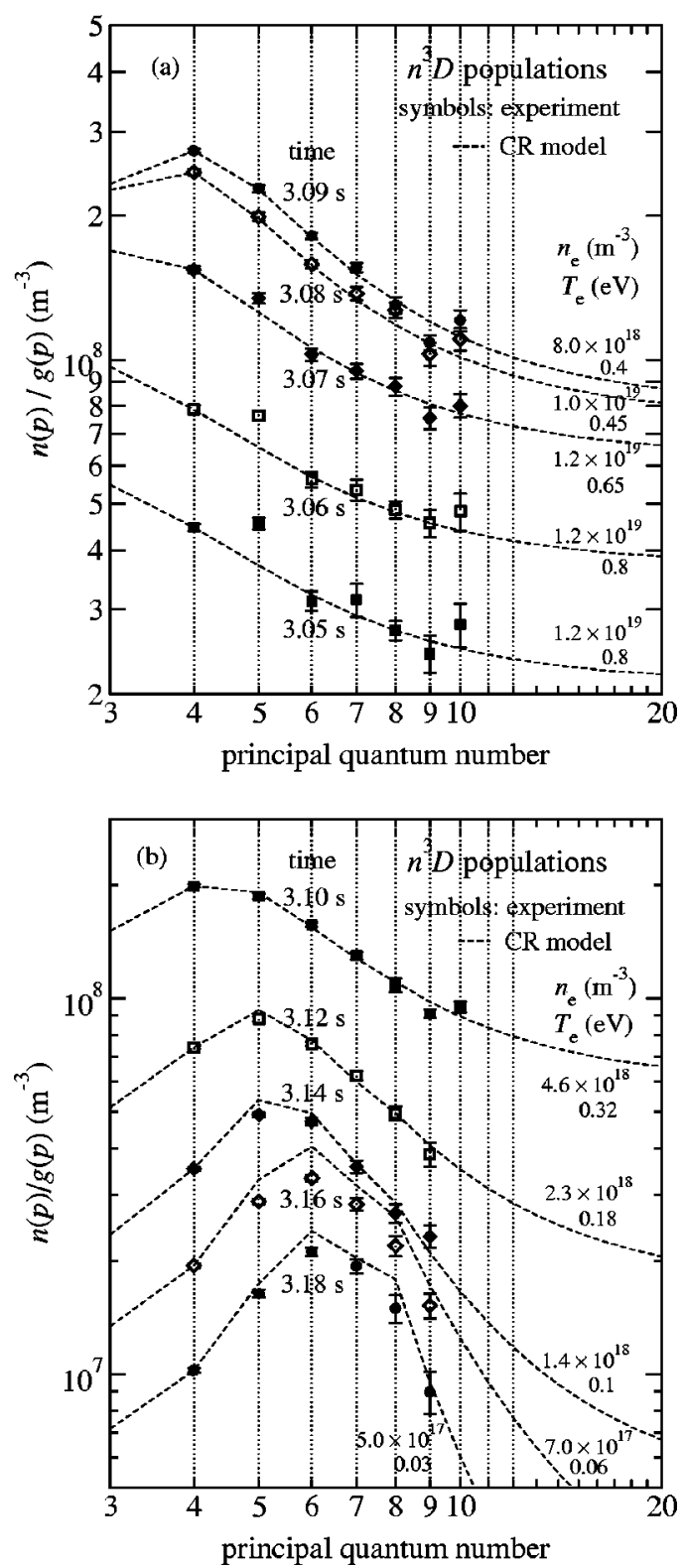

FIG. 8. Similar to Fig. 7, but for the HeI $n^{3} D$ levels in (a) $t$ $=3.05-3.09 \mathrm{~s}$ and (b) $t=3.10-3.18 \mathrm{~s}$. The calculation results are normalized to the experimental data for the $4{ }^{3} D$ level.

calculated populations for $n=8$ is regarded as the contribution from the neutral hydrogen line. The intensity of the $\mathrm{HI} \lambda$ $468.1 \mathrm{~nm}$ line in Fig. 5(a) is thus estimated.

For neutral helium $T_{e}$ and $n_{e}$ are determined in a similar method for the $n^{3} D$ levels $(n \geqslant 4)$. Their populations are deduced from the intensities of the emission lines accompanying the transitions terminating on the $2{ }^{3} P$ level. The determined plasma parameters and the population distribution profile normalized for the level $4{ }^{3} D$ are shown in Fig. 8 . The experimental results of the $n=5$ population in the time range of $t=3.05 \mathrm{~s}-3.07 \mathrm{~s}$ clearly deviate from the calculation results. The difference is found to be as due to the influence from the HeII $(n=4-13)$ line, and hence this level is disregarded in the fitting. The obtained $T_{e}$ and $n_{e}$ from both the neutral and ionized helium are shown in Fig. 9 as a function of time. For the density, in the comparison with the 


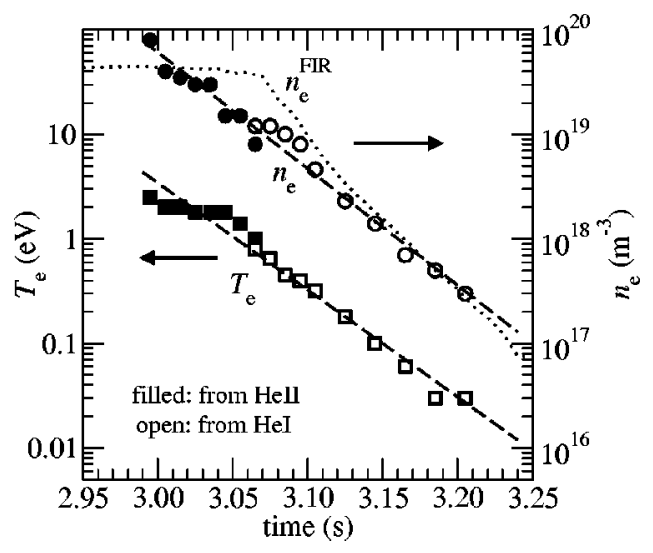

FIG. 9. Temporal variations of $T_{e}$ and $n_{e}$ in the final stage of the discharge as a result of the population calculations in Figs. 7 and 8. Dashed lines are the results of the least squares fitting with an exponential function. Dotted line shows $n_{e}^{F I R}$ which is the same as shown in Fig. 5.

interferometer measurement in Fig. 5, a considerable discrepancy is seen especially in the early time range. We must remember here again that both of the results do not necessarily coincide with each other for the same reason mentioned in Sec. IV A, and for the evaluation of the recombination fluxes our results are appropriate. The data in Fig. 9 are respectively fitted with an exponential function and the results are shown in the same figure with dashed lines.

From the results in Figs. 6 and 9, $\kappa_{0}$ 's are determined for the three emission lines, and with the line intensities in Fig. 5(a) the temporal variations of the individual ion densities are obtained according to Eqs. (12)-(14). The results are shown in Fig. 5(b). The contribution from hydrogen and helium to the total electron density, $\left[n_{e}\right]_{\mathrm{H}, \mathrm{He}}$, is given by

$$
\left[n_{e}\right]_{\mathrm{H}, \mathrm{He}}=n_{\mathrm{H}^{+}}+n_{\mathrm{He}^{+}}+2 n_{\mathrm{He}^{2+}},
$$

and the result is shown in Fig. 5(b). In the same figure $n_{e}^{F I R}$ is also shown. The temporal behavior of $\left[n_{e}\right]_{\mathrm{H}, \mathrm{He}}$ is similar to that of $n_{e}^{F I R}$, and the latter is larger than the former by a factor of 1.5 to 2 . We judge the agreement is satisfactory for the same reasons as given in the preceding section, and some further considerations will be made in the next section. However, it can at least be said that, from the similar decay times of $\left[n_{e}\right]_{\mathrm{H}, \mathrm{He}}$ and $n_{e}^{F I R}$, the decrease in the electron density is dominated not by the particle transport, but by the atomic recombination processes. This is a remarkable feature of helical devices in which the steady-state magnetic field for the plasma confinement is present irrespective of the plasma status.

\section{DETERMINATION OF THE ION DENSITIES}

In LHD, even if the discharges are made only with the gas puffing of hydrogen, helium emission lines are observable as the GDC has been conducted prior to the daily experiment, for example. Helium is thought to have been accumulated on the vacuum vessel wall during the GDC or the preceding experiments with helium gas. However, no quantitative evaluation of the amount of helium in the plasma has been made so far. In Sec. IV B it is found that the temporal decrease of the electron and ion densities in the final stage of

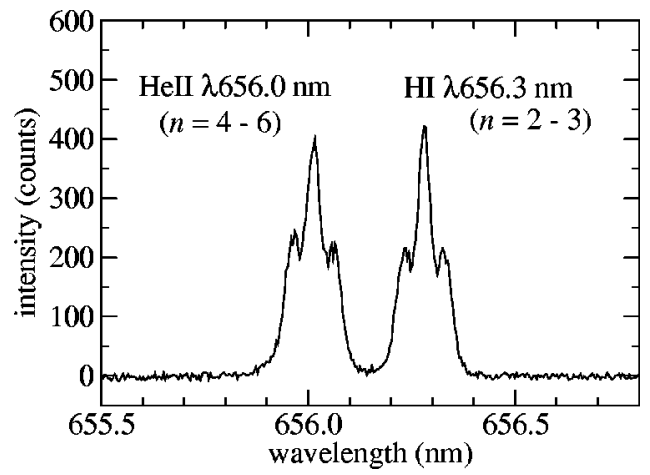

FIG. 10. An example of line profiles for the HI $\lambda 656.3 \mathrm{~nm}$ and HeII $\lambda$ $656.0 \mathrm{~nm}$ lines observed in the final stage of a discharge with the high wavelength resolution measurement.

the discharge is dominated by the recombination processes, and the temporal integration of the recombination flux that is evaluated from the emission line intensity over the recombining phase leads to the ion density just before the plasma termination. Here, we focus on the ion densities for hydrogen and helium and their ratio obtained by this method.

For this purpose it is enough to measure the intensity of one emission line for each of neutral hydrogen and ionized helium since both the ions can be assumed to be fully ionized during the main discharge [see Fig. 5(b)]. However, with the observation system used for the measurement in the preceding sections, it is difficult to determine the line intensities of neutral hydrogen because they are blended with the ionized helium lines. The wavelengths of the two blended lines are slightly different due to the different nuclear mass, and hence they can be separately observed if a sufficiently high wavelength resolution measurement is carried out. Figure 10 shows an example of the line profiles for the HI $\lambda 656.3 \mathrm{~nm}$ and HeII $\lambda 656.0 \mathrm{~nm}(n=4-6)$ lines observed in the final stage of a discharge with a $1.33 \mathrm{~m}$ spectrometer (McPherson Model 209) having a 1800 grooves/mm grating. The viewing chord is the same as the one used in the preceding sections. The lines exhibit Zeeman profiles which indicate the magnetic field strength of about $2.4 \mathrm{~T}$. This field strength corresponds to the location near the plasma center. In this measurement we do not utilize the "fast kinetic mode." Instead the signals of several horizontal lines of pixels on the CCD that covers all of the optical fiber image are summed and are read out periodically. The sampling rate is five frames per second. The recombining plasma is usually observed over two or three frames, and the summation of those signals is regarded as the total intensity in the recombining phase. The coefficient $\kappa_{0}$ for these two lines is shown in Fig. 11. Taking account of the plasma parameters deduced in Sec. IV B, we employ here fixed values for $\kappa_{0}: \kappa_{0}=7$ for neutral hydrogen and $\kappa_{0}=50$ for ionized helium.

Figure 12(a) shows the ratio, $n_{\mathrm{He}^{2+}} /\left(n_{\mathrm{H}^{+}}+n_{\mathrm{He}^{2+}}\right)$, obtained for a series of discharges. For the gas-puffing, helium gas is used for the shot numbers of 30736-30750, and hydrogen gas is used for others. In Fig. 12(b), the $n_{e}^{F I R}$ values just before the plasma termination are shown. It is readily found that helium ions initially predominate over protons even after the fuel gas is changed to hydrogen. The fraction 


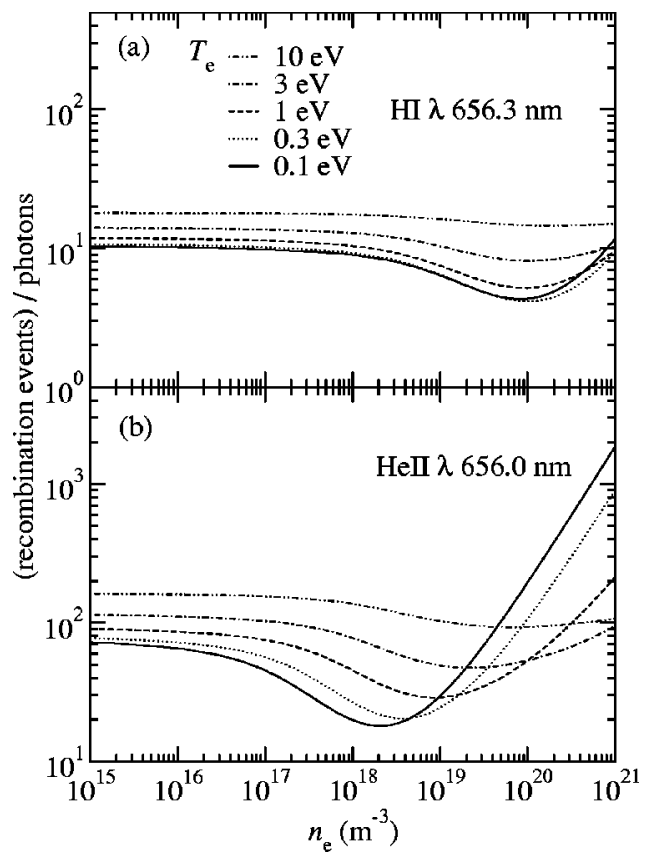

FIG. 11. Dependences of $\kappa_{0}=\alpha_{\mathrm{CR}} / \varepsilon_{0}$ on $T_{e}$ and $n_{e}$ for the (a) $\mathrm{HI} \lambda 656.3$ $\mathrm{nm}$ and (b) HeII $\lambda 656.0 \mathrm{~nm}$ lines.

of helium ions suddenly decreases to about $50 \%$ as $n_{e}^{\text {FIR }}$ increases beyond $5 \times 10^{19} \mathrm{~m}^{-3}$, and this value is kept almost unchanged since then. We have also tried hydrogen ice pellets injection to increase the density, but the situation is completely unchanged. The $n_{e}^{F I R}$ values momentarily reach a peak value of $2 \times 10^{20} \mathrm{~m}^{-3}$ just after the pellet injection or about $1.5 \mathrm{~s}$ before the plasma termination. The fraction of helium ions is probably more reduced at this moment. Even so the remaining part of the discharge and the following

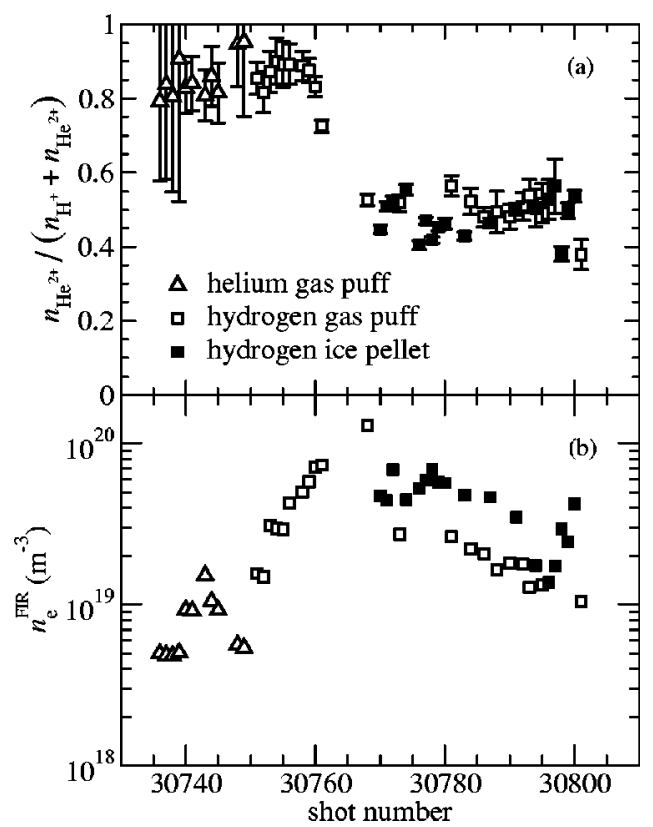

FIG. 12. Variation of (a) the ratio, $n_{\mathrm{He}^{2+}} /\left(n_{\mathrm{H}^{+}}+n_{\mathrm{He}^{2+}}\right)$ and (b) $n_{e}^{F I R}$ for a series of discharges. Open triangles and open and filled squares indicate the discharges with helium gas-puffing, hydrogen gas-puffing, and hydrogen ice pellets, respectively.

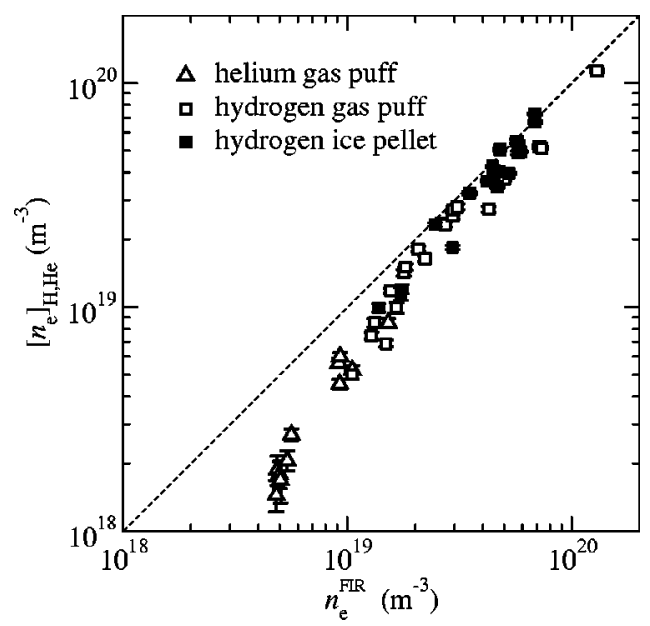

FIG. 13. Dependence of $n_{\mathrm{H}^{+}}+2 n_{\mathrm{He}^{2+}}=\left[n_{e}\right]_{\mathrm{H}, \mathrm{He}}$ on $n_{e}^{F I R}$ for the data in Fig. 12. The symbol definitions are the same as those for Fig. 12.

discharges are unaffected with regard to the abundance of helium ions. It is worth mentioning that when we employ hydrogen gas instead of helium gas for the glow discharge cleaning, it is found that the fraction of helium ions is reduced to be less than $1 \%$ in the subsequent experiment.

The summation, $n_{\mathrm{H}^{+}}+2 n_{\mathrm{He}^{2+}}=\left[n_{e}\right]_{\mathrm{H}, \mathrm{He}}$, represents the contribution of hydrogen and helium to the total electron density. In Fig. 13, $\left[n_{e}\right]_{\mathrm{H}, \mathrm{He}}$ for the results in Fig. 12 is plotted against $n_{e}^{F I R}$. It is seen that the agreement between $\left[n_{e}\right]_{\mathrm{H}, \mathrm{He}}$ and $n_{e}^{F I R}$ is better in high density cases and this suggests that the plasma purity is better for higher density discharges. It should be noted here that the dependences of $\kappa_{0}$ on the plasma parameters are ignored here. According to Fig. 11, they have larger values in the low density range than the fixed ones employed here. This fact could be one of the reasons for the discrepancy in the low density range in Fig. 12. In this case, however, the influence of the parameter dependences of $\kappa_{0}$ is too small to explain it. According to Fig. 13, a half of the electrons originate from the impurities at $n_{e}^{F I R}=1 \times 10^{19} \mathrm{~m}^{-3}$. In order to make the results certain, it would be necessary to determine the amount of impurity ions directly. The present method to determine the ion density of hydrogen and helium is also applicable to the impurity ions.

\section{ACKNOWLEDGMENT}

We wish to thank the entire LHD staff for supporting the experiment.

${ }^{1}$ M. Goto, K. Sawada, and T. Fujimoto, Phys. Plasmas 9, 4316 (2002).

${ }^{2}$ O. Motojima, H. Yamada, A. Komori et al., Phys. Plasmas 6, 1843 (1999).

${ }^{3}$ K. Saito, R. Kumazawa, T. Mutoh et al., Nucl. Fusion 41, 1021 (2001).

${ }^{4}$ K. Sawada, thesis, Kyoto University, 1994.

${ }^{5}$ L. C. Johnson, Astrophys. J. 174, 227 (1972).

${ }^{6}$ T. Fujimoto, J. Quant. Spectrosc. Radiat. Transf. 21, 439 (1979).

${ }^{7}$ M. Goto, J. Quant. Spectrosc. Radiat. Transf. 76, 331 (2003).

${ }^{8}$ D. V. Fursa and I. Bray, Phys. Rev. A 52, 1279 (1995).

${ }^{9}$ I. Bray and D. V. Fursa, J. Phys. B 28, L197 (1995).

${ }^{10}$ D. V. Fursa and I. Bray, J. Phys. B 30, 757 (1997).

${ }^{11}$ Y. V. Ralchenko, R. K. Janev, T. Kato, D. V. Fursa, I. Bray, and F. J. de Heer, NIFS-DATA-59, 2000

${ }^{12}$ N. N. Ljepojevic, R. J. Hutcheon, and R. W. P. McWhirter, J. Phys. B 17, 3057 (1984). 
${ }^{13}$ N. N. Ljepojevic, R. J. Hutcheon, and J. Payne, Comput. Phys. Commun. 44, 157 (1987).

${ }^{14}$ V. I. Fisher, Y. V. Ralchenko, V. A. Bernshtam, A. Goldgirsh, Y. Maron, L. A. Vainshtein, I. Bray, and H. Golten, Phys. Rev. A 55, 329 (1997).

${ }^{15}$ M. Goto and S. Morita, Phys. Rev. E 65, 026401 (2002).

${ }^{16}$ K. Kawahata, K. Tanaka, Y. Ito, A. Ejiri, and S. Okajima, Rev. Sci. Instrum. 70, 707 (1999).

${ }^{17}$ B. Schweer, G. Mank, A. Pospieszczyk, B. Brosda, and B. Pohlmeyer, J. Nucl. Mater. 196-198, 174 (1992)

${ }^{18}$ H. Kubo, M. Goto, H. Takenaga, A. Kumagai, T. Sugie, S. Sakurai, N.
Asakura, S. Higashijima, and A. Sakasai, J. Plasma Fusion Res. 75, 945 (1999).

${ }^{19} \mathrm{~S}$. Morita, M. Goto, T. Morisaki et al., in Proceedings of 26th European Conference on Controlled Fusion and Plasma Physics, Maastricht, 1999, edited by B. Schweer, G. Van Oost, and E. Vietzke (European Physical Society, Petit-Lancy, 1999), Vol. 23J, p. 1321.

${ }^{20}$ T. Morisaki, S. Sakakibara, K. Y. Wawtanabe, H. Yamada, S. Masuzaki, N. Ohyabu, A. Komori, K. Yamazaki, and O. Motojima, Contrib. Plasma Phys. 3-4, 266 (2000).

${ }^{21}$ T. Fujimoto, J. Phys. Soc. Jpn. 49, 1561 (1980). 